\title{
Chronic Cough Is Associated With Long Breaks in Esophageal Peristaltic Integrity on High- resolution Manometry
}

\author{
Michael C Bennett, ${ }^{1}$ Amit Patel, ${ }^{1,3}$ Nitin Sainani, ${ }^{1}$ Dan Wang, ${ }^{4}$ Gregory S Sayuk, ${ }^{1,2}$ and C Prakash Gyawali ${ }^{1 *}$ \\ ${ }^{\prime}$ Division of Gastroenterology, Washington University School of Medicine, St. Louis, MO, USA; ${ }^{2}$ Division of Gastroenterology, John Cochran VA \\ Medical Center Saint Louis, MO, USA; ${ }^{3}$ Division of Gastroenterology, Duke University School of Medicine and the Durham VA Medical Center, \\ Durham, NC, USA; and ${ }^{4}$ Division of Gastroenterology, The First Hospital of Jilin University, Changchung, Jilin, China
}

\section{Background/Aims}

Breaks in the peristaltic contour on esophageal high-resolution manometry (HRM) may be associated with bolus retention in the esophagus. We evaluated the relationship between peristaltic breaks and esophageal symptoms, reflux exposure, and symptom outcomes in a prospective patient cohort.

\section{Methods}

Two hundred and eighteen patients (53.2 \pm 0.9 years, $68.3 \%$ female) undergoing both pH-impedance testing and HRM over a 5 -year period were prospectively evaluated. Demographics, symptom presentation, acid exposure time, symptom association probability, and symptom burden scores were collected. Outcomes were assessed on follow-up using changes in symptom scores. Presence of long breaks $(\geq 5 \mathrm{~cm})$ on HRM was assessed by a blinded author. Relationships between breaks, reflux parameters, presenting symptoms, and outcomes were assessed.

\section{Results}

Patients with long breaks were more likely to have cough as a presenting symptom than those without $(43.4 \%$ vs $28.6 \%, P=$ 0.024); statistical differences were not demonstrated with other symptoms $(P \geq 0.3)$. Numbers of swallows with long breaks were higher in patients with cough compared to those without $(2.4 \pm 0.3$ vs $1.6 \pm 0.2, P=0.021)$; differences were not found with other symptoms $(P \geq 0.4)$. Long breaks were not associated with age, gender, race, reflux burden, symptom association, or changes in symptom metrics ( $P \geq 0.1$ for all comparisons). Among patients with cough, the presence of long breaks predicted suboptimal symptom improvement with antireflux therapy $(P=0.018)$; this difference did not hold true for other symptoms $(P \geq 0.2)$.

\section{Conclusions}

Long breaks in esophageal peristaltic integrity are associated with cough. The presence of long breaks is associated with suboptimal benefit from antireflux therapy.

(J Neurogastroenterol Motil 2018;24:387-394)

Key Words

Cough; Esophageal motility disorders; Esophageal pH monitoring; Gastroesophageal reflux

Received: October 23, 2017 Revised: February 14, 2018 Accepted: March 26, 2018

(c) This is an Open Access article distributed under the terms of the Creative Commons Attribution Non-Commercial License (http://creativecommons. org/licenses/by-nc/4.0) which permits unrestricted non-commercial use, distribution, and reproduction in any medium, provided the original work is properly cited.

${ }^{*}$ Correspondence: C Prakash Gyawali, MD

Division of Gastroenterology, 660 South Euclid Ave., Campus Box 8124, St. Louis, MO 63110, USA

Tel: +1-314-454-8201, Fax: +1-314-454-5107, E-mail: cprakash@dom.wustl.edu 


\section{Introduction}

Gastroesophageal reflux disease (GERD) commonly presents with typical symptoms of heartburn and acid regurgitation, but on occasion, can manifest as atypical symptoms, including chest pain, cough, and laryngeal symptoms. ${ }^{1}$ For instance, GERD is the most common cause of atypical chest pain not due to a cardiac cause, ${ }^{2}$ and is believed to be the third most common cause triggering chronic cough, after post-nasal drip and asthma. ${ }^{3}$ The likelihood of endoscopic evidence of GERD is much lower with atypical symptoms when compared to typical symptoms, ${ }^{4}$ and symptom improvement from acid suppressive therapy can be suboptimal. ${ }^{5}$ Therefore, atypical symptoms often require evaluation with esophageal physiologic tests including ambulatory $\mathrm{pH}$ or $\mathrm{pH}$-impedance monitoring (typically performed off antisecretory therapy in the setting of unproven GERD), and esophageal high-resolution manometry (HRM). ${ }^{1}$

While it is well known that esophageal hypomotility patterns are associated with more profound esophageal reflux burden, ${ }^{6}$ only limited evidence points towards association of such patterns with specific GERD symptoms. In particular, the presence of weak peristalsis with long breaks has been shown to be more prevalent in patients with chronic cough when compared to heartburn, with evidence of incomplete bolus transit on stationary impedance in the setting of breaks. ${ }^{7}$ The relationship of peristaltic breaks with esophageal symptoms is intriguing, since breaks could potentially allow for prolonged retention of bolus or refluxate, and the proximal esophagus is known to be more sensitive to bolus presence. ${ }^{8}$ However, the associations between symptoms, peristaltic breaks, and symptomatic outcomes with antireflux therapy remain incompletely understood.

In this study, we evaluated peristaltic breaks on HRM in a prospective cohort of patients with reflux symptoms undergoing $\mathrm{pH}$-impedance testing, where reflux burden was well characterized, and symptom-reflux association was documented. Our aims were: (1) to assess the prevalence of abnormal motor patterns, particularly breaks in the peristaltic contour, within patients with typical and atypical reflux symptoms, (2) to evaluate relationships between specific esophageal symptoms, reflux burden, and peristaltic breaks on HRM, and (3) to determine if the presence of peristaltic breaks predict symptom outcomes with antireflux therapy.

\section{Materials and Methods}

\section{Subjects}

All adult patients ( $\geq 18$ years) with persistent GERD symptoms despite anti-secretory therapy referred to the Gastroenterology Motility Center at Washington University in Saint Louis for ambulatory $\mathrm{pH}$-impedance testing and HRM from January 2005 through August 2010 were eligible for inclusion. Exclusion criteria included inadequate studies (poor data quality precluding analysis), incomplete studies ( $<14$ hours recording time for $\mathrm{pH}$-impedance, inadequate swallow complement on HRM), presence of major esophageal motor disorders (achalasia spectrum disorders, socalled "scleroderma esophagus"), and history of fundoplication or other foregut surgery. Patients were identified from our institution's database and contacted for informed consent; they were prospectively followed up and repeat symptom assessment was performed by questionnaire. Patient management was not influenced by this study protocol; instead, management was directed by the referring physicians. The data-set from which this analysis was performed has been previously utilized in analyzing GERD phenotypes, and in identifying metrics from $\mathrm{pH}$-impedance testing that predict GERD symptom response. ${ }^{10,11}$ This study protocol was approved by the Human Research Protection Office (institutional review board) at Washington University in Saint Louis (201105490 approved 6/6/2011). Informed consent was obtained from each patient for collection and analysis of HRM, and $\mathrm{pH}$-impedance and symptom questionnaire data.

\section{High-resolution Manometry}

HRM studies were performed after an overnight fast using a 36-channel solid-state catheter system with high-fidelity circumferential sensors $1 \mathrm{~cm}$ apart along the catheter (Covidien/Given Imaging, Duluth, GA, USA). After calibration, the catheter was passed through an anesthetized nasal canal. A 20-second swallow-free period was obtained while the subject remained still, resting quietly in the recumbent position (landmark period) from which basal pressures were obtained. ${ }^{12}$ Ten swallows were recorded using 4-5 mL of ambient temperature water spaced $>20$ seconds apart. Studies were acquired and analyzed using dedicated computerized HRM acquisition, display, and analysis systems (ManoView; Covidien/ Given Imaging). A blinded author reviewed HRM studies for presence and length of breaks in the peristaltic contour of individual swallows, using the $20 \mathrm{~mm}$ isobaric contour tool. Since this study 
was designed to evaluate the role of long breaks, any presence of breaks, and particularly breaks $\geq 5 \mathrm{~cm}$ were recorded (in contrast to the Chicago classification v3.0 where breaks are not evaluated if distal contractile integral (DCI) $<450 \mathrm{mmHg} \cdot \mathrm{cm} \cdot \mathrm{sec}) .{ }^{13}$ Swallows with long breaks (breaks $\geq 5 \mathrm{~cm}$ in length) were designated as either fragmented (DCI $>450 \mathrm{mmHg} \cdot \mathrm{cm} \cdot \mathrm{sec}$ ) or ineffective (DCI $<450 \mathrm{mmHg} \cdot \mathrm{cm} \cdot \mathrm{sec}$ ) according to the Chicago Classification v3.0 (Fig. 1). ${ }^{13}$

\section{pH-Impedance Monitoring}

pH-impedance testing was offered in an "open-access" fashion such that referring physicians decided whether testing was performed on or off antisecretory therapy. Patients tested off therapy were asked to stop their proton pump inhibitor (PPI) medications 5-7 days prior to the study, and histamine-2 receptor antagonists, prokinetic medications, and antacids 3 days prior to the study. After an overnight fast, an experienced nurse positioned the $\mathrm{pH}$ impedance catheter (Sandhill Scientific, Highlands Ranch, CO, USA) with the distal esophageal $\mathrm{pH}$ sensor placed $5 \mathrm{~cm}$ proximal to the lower esophageal sphincter, measured using HRM. During the data acquisition period, patients recorded meals and activities on a paper diary and logged symptom events electronically using the device data recorder. Data were then analyzed with dedicated software (Bioview Analysis, Sandhill Scientific, Highlands Ranch, CO, USA) with automated calculation of acid exposure time (AET), reflux episodes, symptom events, and symptom-reflux association parameters. Each pH-impedance study was scrutinized manually by 2 reviewers (Amit Patel and C Prakash Gyawali) to ensure the accuracy of automated capture of reflux events; any discrepancies between the reviewers were resolved by discussion.

Since study patients had $\mathrm{pH}$-impedance studies performed both on and off PPI therapy, symptom reflux correlation was first assessed. Symptoms were considered associated with reflux events if they occurred within 2 minutes following a reflux event. Symptom associated probability (SAP) was calculated using the Ghillebert probability estimate. ${ }^{14} \mathrm{SAP}$ was designated positive if the likelihood of a chance association between symptoms and reflux events was $<$ $5 \%$, corresponding to $P<0.05$ and $\mathrm{SAP}>95 \%$. The SAP was first calculated for $\mathrm{pH}$-detected reflux events, then re-calculated for all impedance-detected reflux events. Our group has previously shown that Ghillebert probability estimate correlates very well with SAP calculated using the Weusten method, with sensitivity of 0.95 and specificity of $0.91 .^{15,16}$ AET was also recorded, calculated as the percentage of time the $\mathrm{pH}$ was below 4 at the distal esophageal $\mathrm{pH}$ sensor. $^{10,17}$

\section{Symptom Burden}

Patients reported symptom burden prior to $\mathrm{pH}$-impedance monitoring both for dominant presenting symptom and global esophageal symptoms. Symptom frequency and severity were rated on 5-point Likert scales generated a priori for esophageal testing at our center and validated for assessment of esophageal symptom. ${ }^{10,11,18,19}$ For each esophageal symptom, patients rated symptom frequency from 0 (no symptoms) to 4 (very severe symptoms); the product of severity and frequency yielded a total score from 0 to 16 . We have previously reported this as dominant symptom intensity. ${ }^{10,11}$ However, for the purpose of this study, symptom intensity was calculated for each esophageal symptom reported by the patients, re-
A

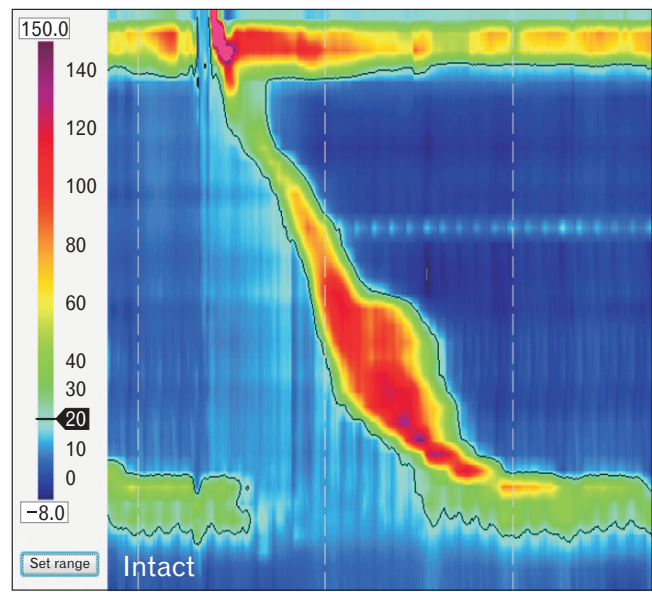

B

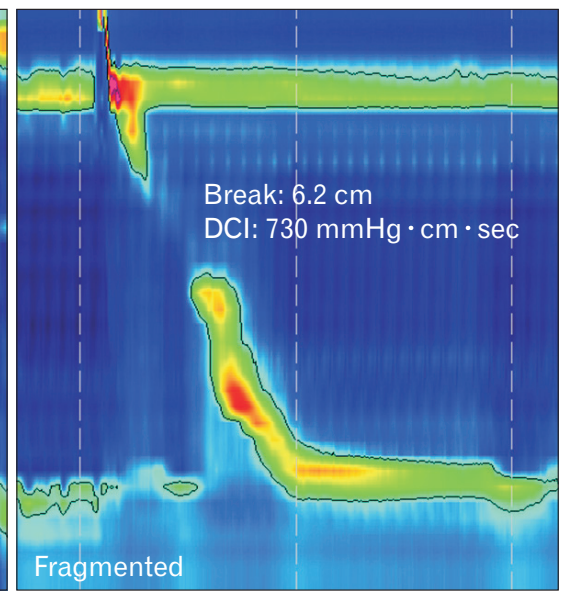

C

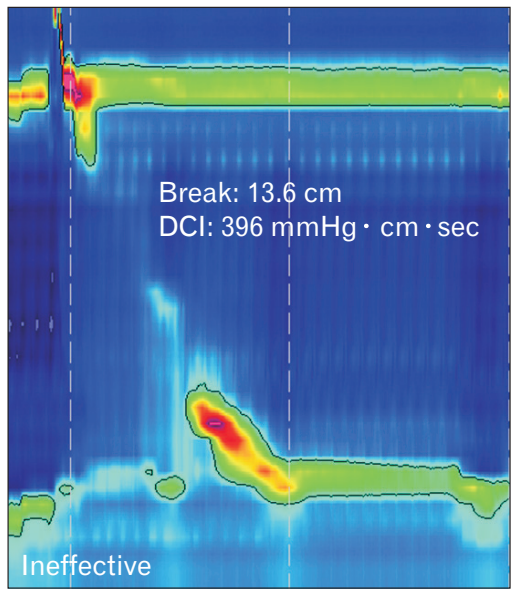

Figure 1. High-resolution manometry Clouse plots demonstrating (A) intact peristaltic contour in an effective swallow, (B) fragmented sequence with a long $(>5 \mathrm{~cm})$ break, and $(\mathrm{C})$ ineffective sequence with a long break. 
gardless of whether the symptom was dominant or secondary, hence this is termed esophageal symptom intensity (ESI). Patients rated their global symptom severity (GSS) pertaining to the esophagus over the previous 2 weeks on a $100-\mathrm{mm}$ visual analog scale. Basic demographic data (age, gender, and race) were also collected.

Eligible subjects were prospectively contacted to assess management approaches (surgical versus medical therapy) and symptomatic outcomes, and post-therapy symptom questionnaire data was required for evaluation of treatment outcome. The pre-procedure symptom survey was re-administered at this follow-up, and linear as well as dichotomous ( $\geq 50 \%$ ) improvement in ESI and GSS were calculated to quantify symptomatic outcomes. Each patient's treating physician guided management with no influence from study investigators.

\section{Statistical Methods}

Data are reported as the mean \pm standard error of the mean unless otherwise indicated. Demographics, PPI status on testing, baseline symptom burden (ESI and GSS), change in symptom burden with treatment, SAP, and presence of specific reflux symptoms (heartburn, regurgitation, chest pain, cough, dysphagia, and laryngeal symptoms) were compared between patients with and without long breaks in the peristaltic contour. Categorical data were compared using the $\chi$-squared test, and continuous data were compared using ANOVA, 2-tailed Student's t test, or Mann-Whitney $\mathrm{U}$ test as appropriate. In all cases, $P<0.05$ was required for statistical significance. All statistical analyses were performed using IBM SPSS Statistics V.22.0 (Armonk, NY, USA).

\section{Results}

Of 302 patients undergoing HRM in conjunction with ambulatory $\mathrm{pH}$-impedance monitoring for GERD symptoms, inclusion criteria were met by 218 patients (53.2 \pm 0.9 years, $68.3 \%$ female; Fig. 2). Presenting symptoms included both typical symptoms (heartburn: $64.2 \%$, regurgitation: $48.6 \%$ ), and atypical symptoms (chest pain: $30.7 \%$, cough: $35.8 \%$, dysphagia: $17.4 \%$, laryngeal symptoms: $11.0 \%) ; 168$ patients $(77.1 \%)$ reported $>1$ symptom. When dominant symptoms were segregated into typical (123 $[56.4 \%])$ and atypical groups (95 [43.6\%]), baseline ESI was higher in atypical presentations $(P=0.012$; Table 1$)$ but global symptom burden was similar between these 2 groups $(P=0.243)$; AET was higher with typical presentations $(P=0.022)$. There were no significant differences in demographic, motor diagnoses or $\mathrm{pH}$ impedance metrics between patients with and without long breaks ( $P>0.1$ for all comparisons); in particular, we did not demonstrate differences in symptom reflux association metrics between patients with and without long breaks.

The relationships between individual presenting symptoms and peristaltic breaks were further assessed. Cough was seen more often as a presenting symptom in patients tested off PPI therapy $(48 / 111$, $43.2 \%)$ compared to those tested on therapy $(30 / 107,28 \% ; P=$ 0.020). Patients with cough tested off PPI had higher total acid exposure times compared to those tested on PPI ( $5.3 \pm 0.8$ vs $0.9 \pm$ $0.3, P<0.001)$. Despite this, overall symptom burden was similar between patients tested off vs on PPI therapy (ESI: $9.3 \pm 0.6$ vs 8.8
$302 \mathrm{pH}$-impedance studies in 5 -year study period
84 Patients ineligible

7 incomplete $\mathrm{pH}$-impedance studies

48 incomplete HRM studies

3 motor disorders

26 prior surgery

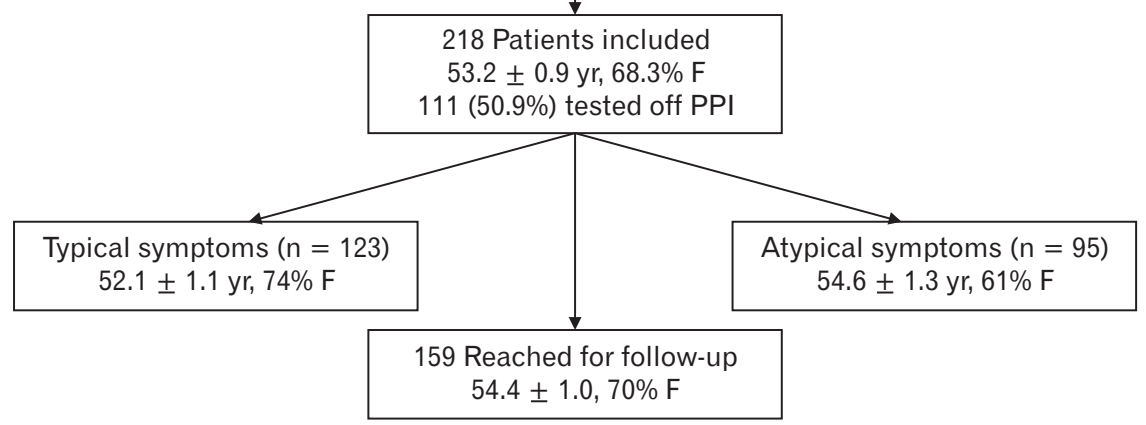

Figure 2. Flow chart describing the study population. HRM, high-resolution manometry; F, female; PPI, proton pump inhibitor. 
Table 1. Comparison of Clinical Characteristics Between Typical and Atypical Symptoms

\begin{tabular}{lccc}
\hline \multicolumn{1}{c}{ Clinical characteristics } & $\begin{array}{c}\text { Typical } \\
\text { symptoms } \\
(\mathrm{n}=123)\end{array}$ & $\begin{array}{c}\text { Atypical } \\
\text { symptoms } \\
(\mathrm{n}=95)\end{array}$ & $P$-values \\
\hline Age (yr) & $52.1 \pm 1.1$ & $54.6 \pm 1.3$ & 0.151 \\
Gender (\% female) & 74 & 61 & 0.056 \\
Baseline ESI & $8.1 \pm 0.6$ & $10.3 \pm 0.7$ & 0.011 \\
Baseline GSS & $60.8 \pm 2.7$ & $65.7 \pm 3.1$ & 0.243 \\
AET total (\%) & $4.4 \pm 0.5$ & $2.8 \pm 0.4$ & 0.022 \\
IEM & $37(30 \%)$ & $18(19 \%)$ & 0.507 \\
Fragmented peristalsis & $1(1 \%)$ & $1(1 \%)$ & $>0.99$ \\
Normal peristalsis & $85(69 \%)$ & $72(76 \%)$ & 0.291 \\
Swallows with long breaks & $2.0 \pm 0.3$ & $1.7 \pm 0.3$ & 0.313 \\
\hline ESI, eso & & &
\end{tabular}

ESI, esophageal symptom intensity; GSS, global symptom severity; AET, acid exposure time; IEM, ineffective esophageal motility.

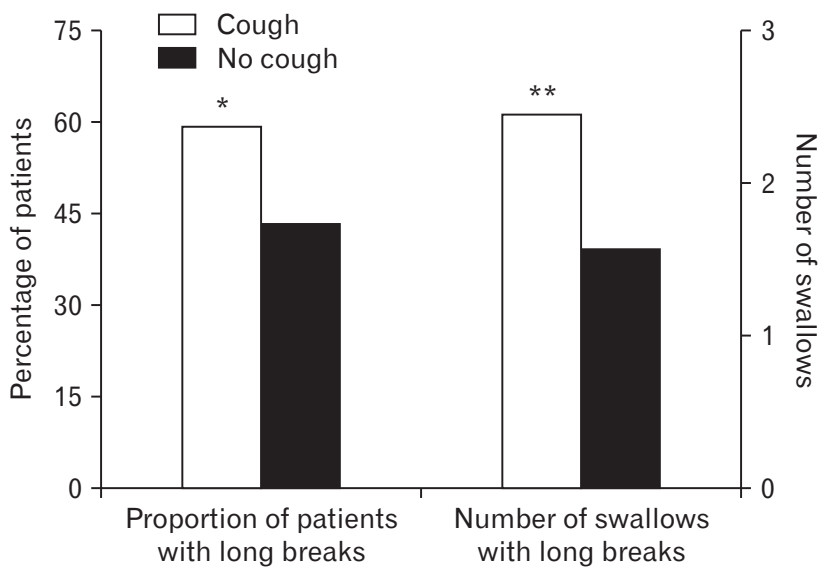

Figure 3. Proportion of breaks in patients with cough. More patients with cough had long $(>5 \mathrm{~cm}$ ) breaks in the peristaltic contour compared to patients without cough $\left({ }^{\star} P=0.024\right)$. Numbers of swallows with breaks was also significantly higher in patients with cough $\left({ }^{* *} P=\right.$ $0.021)$.

\pm 0.6 respectively, GSS: $62.6 \pm 3.0$ vs $63.3 \pm 2.8$ respectively, $P$ $>0.6$ for each comparison). Further, PPI status had no bearing on esophageal motor pattern based on the Chicago classification v3.0 ( $P$ $>0.8$ for each comparison).

Patients with cough were significantly more likely than those without cough to have long breaks in the peristaltic contour $(59.0 \%$ vs $42.8 \%, P=0.024$; Fig. 3 ); this did not differ whether testing was performed off or on PPI status. The odds ratio of cough as a presenting symptom in the setting of breaks was 1.9 (95\% confidence interval: 1.1-3.4, $P=0.023$ ); none of the other presenting symptoms were similarly associated (odds ratios $0.73-1.03, P \geq$ 0.3 ). The average number of swallows with long breaks was higher
Table 2. Comparison of Clinical Characteristics Between Patients With and Without Cough

\begin{tabular}{lccc}
\hline \multicolumn{1}{c}{ Clinical characteristics } & $\begin{array}{c}\text { Cough } \\
(\mathrm{n}=78)\end{array}$ & $\begin{array}{c}\text { No cough } \\
(\mathrm{n}=140)\end{array}$ & $P$-values \\
\hline Age (yr) & $51.3 \pm 1.1$ & $56.7 \pm 1.4$ & 0.002 \\
Gender (\% female) & 67 & 69 & 0.762 \\
Baseline ESI & $10.5 \pm 0.7$ & $8.3 \pm 0.5$ & 0.018 \\
Baseline GSS & $67.3 \pm 3.5$ & $60.4 \pm 2.5$ & 0.111 \\
AET total $(\%)$ & $3.6 \pm 0.5$ & $3.7 \pm 0.5$ & 0.892 \\
IEM & $24(31 \%)$ & $35(25 \%)$ & 0.427 \\
Fragmented peristalsis & $1(1 \%)$ & $1(1 \%)$ & $>0.99$ \\
Normal peristalsis & $53(68 \%)$ & $100(71 \%)$ & 0.644 \\
Swallows with long breaks & $2.4 \pm 0.3$ & $1.6 \pm 0.2$ & 0.031 \\
\hline
\end{tabular}

ESI, esophageal symptom intensity; GSS, global symptom severity; AET, acid exposure time; IEM, ineffective esophageal motility.



Figure 4. Predictors of symptom change from antireflux therapy. On multivariate linear regression, the presence of long breaks was a predictor of suboptimal symptom outcome from antireflux therapy in patients with cough $\left({ }^{*} P=0.010\right)$. ESI, esophageal symptom intensity; PPI, proton pump inhibitor; AET, acid exposure time; SAP, symptom association probability.

in patients with cough compared to patients without cough $(2.4 \pm$ 0.3 vs $1.6 \pm 0.2, P=0.021$; Fig. 3 ). Although the average length of peristaltic breaks was similar between patients presenting with and without cough $(7.6 \pm 0.5 \mathrm{~cm}$ vs $8.1 \pm 0.4 \mathrm{~cm}, P=0.496)$, the likelihood of fragmented sequences were higher with cough $(21.8 \%$ vs $10.7 \%, P=0.044$ ). Despite this, the proportion of patients classified with ineffective esophageal motility (IEM) was similar. None of other typical or atypical presenting symptom was associated with breaks in this fashion. Mean DCI, numbers of ineffective swallows, and proportions with IEM were similar regardless of presenting symptom. Cough patients were slightly older and had higher baseline symptom burden, but were otherwise similar to patients without cough (Table 2). 


\section{Symptom Outcomes}

One hundred and fifty-nine patients were successfully contacted to assess symptom outcome after $36.1 \pm 1.2$ months. Overall, ESI decreased by $5.3 \pm 0.5$ and GSS by $35.2 \pm 2.8$. Though patients with atypical symptoms had a higher baseline ESI than those with typical symptoms $(10.3 \pm 0.7$ vs $8.1 \pm 0.6, P=0.012)$, GSS was similar between the groups, and there was no difference in degree of improvement in symptom burden with treatment. The presence or absence of long breaks did not predict symptom burden or change in symptoms in the overall cohort.

Independent of reflux parameters, the presence of long breaks with cough as a presenting symptom was a negative predictor for ESI change with antireflux therapy (ESI improvement of $4.9 \pm$ 0.9) compared to patients without long breaks (ESI improvement of $8.9 \pm 1.4, P=0.018)$. GSS improvement was similar regardless of the presence of breaks $(39.9 \pm 5.8$ vs $44.6 \pm 8.4$ respectively, $P$ $=0.639)$. The presence or absence of long breaks did not predict improvement in ESI or GSS in a similar fashion with presenting symptoms other than cough ( $P \geq 0.3$ for each comparison).

A multivariate regression model was generated using the presence of long breaks, age, gender, PPI status, abnormal AET, and positive SAP as independent variables, and changes in ESI and GSS as the outcome variables. In this model, long breaks retained significance as an independent predictor of suboptimal treatment outcome within the cough cohort following antireflux therapy, by ESI but not GSS (Fig. 4). This significance did not hold true for any other typical or atypical reflux symptoms $(P \geq 0.2$ for each comparison).

\section{Discussion}

In this study, we demonstrate that patients with long breaks in the esophageal peristaltic contour on HRM were more likely to have cough as a presenting symptom compared to other typical and atypical symptoms. Conversely, patients with cough were more likely to have swallows with long breaks in the peristaltic contour, and had a higher number of swallows with breaks. These findings were independent of esophageal reflux burden, numbers of reflux events, or symptom-reflux association with cough events on ambulatory reflux monitoring. Our findings validate the report by Almansa et $\mathrm{al}^{7}$ that weak peristalsis with long breaks is encountered with chronic cough; we further report that this association is not replicated with other typical or atypical symptoms evaluated with $\mathrm{pH}$-impedance monitoring. Finally, our findings suggest that patients with cough and long breaks have worse symptomatic outcomes with antireflux therapy compared to patients with cough without long breaks, or patients with other esophageal symptoms, with or without long breaks.

The significance of breaks in the peristaltic contour on esophageal HRM has been extensively debated. The presence of breaks on combined HRM and impedance testing has been reported to be associated with dysphagia and incomplete bolus clearance ${ }^{20,21}$; in contrast, small breaks are associated with complete bolus clearance and are of questionable clinical significance. ${ }^{22}$ Weak peristalsis with long breaks is reported to be associated with increased acid exposure and delayed reflux clearance. ${ }^{6}$ Although the Chicago classification v3.0 recognizes long breaks only in the context of DCI > $450 \mathrm{mmHg} \cdot \mathrm{cm} \cdot \mathrm{sec}$ (fragmented sequences), ${ }^{13}$ there is evidence to suggest that bolus transit is incomplete with long breaks in general, regardless of vigor of remnant contraction in the esophagus. ${ }^{7,21}$ Both fragmented and ineffective sequences (where DCI $<450$ $\mathrm{mmHg} \cdot \mathrm{cm} \cdot \mathrm{sec}$ regardless of breaks) are associated with GERD in general, and especially more profound manifestations of GERD such as Barrett's esophagus. ${ }^{23,24}$ While the Chicago classification does not have an IEM category with long breaks, Almansa et $\mathrm{al}^{7}$ report that this phenotype may be associated with abnormal bolus clearance, likely from bolus escape through the break. Our findings therefore add to the growing body of evidence supporting a link between long breaks and esophageal symptoms, particularly in cough as a presenting symptom. ${ }^{6,7}$

The precise mechanism of symptom generation from reflux of gastric content is unclear. Both chemical and mechanical receptors participate in reflux symptom generation, especially heartburn and chest pain. Cough in particular is thought to have 2 separate mechanisms leading to symptom generation-aspiration of refluxate into the trachea (likely infrequent), and triggering of a reflexive cough from esophageal stimulation from the refluxate (likely more prevalent). Abnormal upper esophageal sphincter function and esophageal dysmotility have been correlated with chronic cough and positive symptom association in cough patients. ${ }^{25}$ Using $\mathrm{pH}$ impedance monitoring, more proximal migration of reflux events has been found to be associated with higher perception of heartburn and regurgitation. ${ }^{26,27}$ Afferent sensory nerve endings have been demonstrated to be more superficially located in the proximal esophagus, ${ }^{8}$ potentially explaining higher perception in the precise area where we report long breaks in the peristaltic contour. Peristaltic defects in the transition zone between the proximal and distal esophagus are associated with impaired bolus clearance and are common regardless of whether IEM is confirmed. ${ }^{28}$ Therefore, it is 
possible that breaks are associated with prolonged refluxate contact from incomplete reflux clearance in the more perceptive proximal esophagus, leading to cough as a symptom. We demonstrate that cough patients with breaks in the peristaltic contour have a less robust response to antireflux therapy, potentially related to inability to clear refluxate despite reduced acid exposure. Our study was not designed to evaluate these proposed mechanisms for symptom generation, but our findings support a higher likelihood of lack of contractile activity in the proximal and mid esophagus in patients with cough.

The current study has a few important limitations. Patients included in this cohort were identified retrospectively from the original prospectively collected database, and therefore, the study is subject to limitations associated with retrospective studies. We do not have data on the nature of evaluation of chronic cough, and whether all alternate possibilities were rigorously excluded prior to esophageal physiologic testing. Symptom-reflux association with cough as a presenting symptom is difficult, since patients may not activate event monitor buttons in a timely fashion, particularly when cough is frequent or paroxysmal ${ }^{29}$; a cough event monitor was not utilized in this study. Patient management was directed by treating physicians, and therefore there was no standard protocol employed for GERD management, which could have affected our outcome data. We also do not have post treatment esophageal function testing to determine if breaks resolved or if $\mathrm{pH}$ impedance metrics changed in responders in contrast to non-responders. Our patient population consists of individuals evaluated at a tertiary care center, and therefore our results may not be generalizable to primary care populations. Nevertheless, our findings are in concert with prior reports linking long breaks to cough as a presenting symptom in the setting of reflux evaluation, and therefore support further research into understanding the relationship between esophageal symptoms and motor patterns.

We conclude that analyzing esophageal motor patterns beyond what Chicago classification v3.0 encompasses has clinical utility, particularly in the prediction of symptom response in patients with cough. What is evident from our findings and other studies in the literature is that cough is a complex symptom with links to esophageal motor function. Despite this, treating concurrent reflux does not appear sufficient to resolve this symptom. While the precise pathophysiologic basis of the association between cough and long breaks is unclear, existing esophageal pathophysiologic data may imply impaired reflux clearance in triggering symptoms. Further prospective research evaluating esophageal symptom generation in the context of typical and atypical reflux symptoms is needed.
Acknowlegements: This study has been presented as a podium presentation at the Annual Meeting of the American Gastroenterological Association San Diego, CA, USA, May 2016.

Financial support: This study was partially funded through NIH/ NIDDK (T32 DK007130: Amit Patel; NIH K23DK84413-4: Gregory S Sayuk), and through the Washington University Department of Medicine Mentors in Medicine (MIM) and Clinical Science Training and Research (CSTAR) programs.

Conflicts of interest: $\mathrm{C}$ Prakash Gyawali has received research funding and speaking fees from Medtronic, speaking fees from Allergan, and consultation fees from Torax, Quintiles, and Ironwood. None of the other authors have any disclosures. No conflicts of interest exist. No writing assistance was obtained.

Author contributions: Michael C Bennett: study design, data collection and analysis, and manuscript preparation and review; Amit Patel: study design, data analysis, and critical review of manuscript; Nitin Sain and Dan Wang: data collection and critical review of manuscript; Gregory S Sayuk: study design, data analysis, and critical review of manuscript; and C Prakash Gyawali: study concept and design, data analysis, manuscript preparation, critical review, and final approval of manuscript.

\section{References}

1. Katz PO, Gerson LB, Vela MF. Guidelines for the diagnosis and management of gastroesophageal reflux disease. Am J Gastroenterol 2013; 108:308-328.

2. Fass R, Achem SR. Noncardiac chest pain: epidemiology, natural course and pathogenesis. J Neurogastroenterol Motil 2011;17:110-123.

3. Pratter MR. Overview of common causes of chronic cough: ACCP evidence-based clinical practice guidelines. Chest 2006;129(1 suppl):59s$62 \mathrm{~s}$.

4. Dickman R, Mattek N, Holub J, Peters D, Fass R. Prevalence of upper gastrointestinal tract findings in patients with noncardiac chest pain versus those with gastroesophageal reflux disease (GERD)-related symptoms: results from a national endoscopic database. Am J Gastroenterol 2007;102:1173-1179.

5. Dickman R, Boaz M, Aizic S, Beniashvili Z, Fass R, Niv Y. Comparison of clinical characteristics of patients with gastroesophageal reflux disease who failed proton pump inhibitor therapy versus those who fully responded. J Neurogastroenterol Motil 2011;17:387-394.

6. Ribolsi M, Balestrieri P, Emerenziani S, Guarino MP, Cicala M. Weak peristalsis with large breaks is associated with higher acid exposure and delayed reflux clearance in the supine position in GERD patients. Am J Gastroenterol 2014;109:46-51.

7. Almansa C, Smith JA, Morris J, et al. Weak peristalsis with large breaks 
in chronic cough: association with poor esophageal clearance. Neurogastroenterol Motil 2015;27:431-442.

8. Woodland P, Aktar R, Mthunzi E, et al. Distinct afferent innervation patterns within the human proximal and distal esophageal mucosa. Am J Physiol Gastrointest Liver Physiol 2015;308:G525-G531.

9. Patel A, Sayuk GS, Kushnir VM, Chan WW, Gyawali CP. GERD phenotypes from $\mathrm{pH}$-impedance monitoring predict symptomatic outcomes on prospective evaluation. Neurogastroenterol Motil 2016;28:513-521.

10. Patel A, Sayuk GS, Gyawali CP. Parameters on esophageal pH-impedance monitoring that predict outcomes of patients with gastroesophageal reflux disease. Clin Gastroenterol Hepatol 2015;13:884-891.

11. Patel A, Sayuk GS, Gyawali CP. Acid-based parameters on $\mathrm{pH}$-impedance testing predict symptom improvement with medical management better than impedance parameters. Am J Gastroenterol 2014;109:836844.

12. Patel A, Ding A, Mirza F, Gyawali CP. Optimizing the high-resolution manometry (HRM) study protocol. Neurogastroenterol Motil 2015;27: 300-304.

13. Kahrilas PJ, Bredenoord AJ, Fox M, et al. The Chicago Classification of esophageal motility disorders, v3.0. Neurogastroenterol Motil 2015;27: 160-174.

14. Ghillebert G, Janssens J, Vantrappen G, Nevens F, Piessens J. Ambulatory 24 hour intraoesophageal $\mathrm{pH}$ and pressure recordings $\mathrm{v}$ provocation tests in the diagnosis of chest pain of oesophageal origin. Gut 1990;31: 738-744.

15. Weusten BL, Roelofs JM, Akkermans LM, Van Berge-Henegouwen GP, Smout AJ. The symptom-association probability: an improved method for symptom analysis of 24-hour esophageal $\mathrm{pH}$ data. Gastroenterology 1994;107:1741-1745.

16. Kushnir VM, Sathyamurthy A, Drapekin J, Gaddam S, Sayuk GS, Gyawali CP. Assessment of concordance of symptom reflux association tests in ambulatory pH monitoring. Aliment Pharmacol Ther 2012;35: 1080-1087.

17. Kahrilas PJ, Quigley EM. Clinical esophageal $\mathrm{pH}$ recording: a technical review for practice guideline development. Gastroenterology 1996; 110:1982-1996.

18. Kushnir VM, Prakash Gyawali C. High resolution manometry patterns distinguish acid sensitivity in non-cardiac chest pain. Neurogastroenterol
Motil 2011;23:1066-1072.

19. Shaker A, Stoikes N, Drapekin J, Kushnir V, Brunt LM, Gyawali CP. Multiple rapid swallow responses during esophageal high-resolution manometry reflect esophageal body peristaltic reserve. Am J Gastroenterol 2013;108:1706-1712.

20. Bulsiewicz WJ, Kahrilas PJ, Kwiatek MA, Ghosh SK, Meek A, Pandolfino JE. Esophageal pressure topography criteria indicative of incomplete bolus clearance: a study using high-resolution impedance manometry. Am J Gastroenterol 2009;104:2721-2728.

21. Roman S, Lin Z, Kwiatek MA, Pandolfino JE, Kahrilas PJ. Weak peristalsis in esophageal pressure topography: classification and association with dysphagia. Am J Gastroenterol 2011;106:349-456.

22. Park EJ, Lee JS, Lee TH, et al. High-resolution impedance manometry criteria in the sitting position indicative of incomplete bolus clearance. J Neurogastroenterol Motil 2014;20:491-496.

23. Porter RF, Kumar N, Drapekin JE, Gyawali CP. Fragmented esophageal smooth muscle contraction segments on high resolution manometry: a marker of esophageal hypomotility. Neurogastroenterol Motil 2012;24:763-768, e353.

24. Mello MD, Shriver AR, Li Y, Patel A, Gyawali CP. Ineffective esophageal motility phenotypes following fundoplication in gastroesophageal reflux disease. Neurogastroenterol Motil 2016;28:292-298.

25. Vardar R, Sweis R, Anggiansah A, Wong T, Fox MR. Upper esophageal sphincter and esophageal motility in patients with chronic cough and reflux: assessment by high-resolution manometry. Dis Esophagus 2013;26:219-225.

26. Bredenoord AJ, Weusten BL, Curvers WL, Timmer R, Smout AJ. Determinants of perception of heartburn and regurgitation. Gut 2006; 55:313-318.

27. Zerbib F, Duriez A, Roman S, Capdepont M, Mion F. Determinants of gastro-oesophageal reflux perception in patients with persistent symptoms despite proton pump inhibitors. Gut 2008;57:156-160.

28. Xiao Y, Kahrilas PJ, Kwasny MJ, et al. High-resolution manometry correlates of ineffective esophageal motility. Am J Gastroenterol 2012; 107:1647-1654.

29. Kavitt RT, Higginbotham T, Slaughter JC, et al. Symptom reports are not reliable during ambulatory reflux monitoring. Am J Gastroenterol 2012;107:1826-1832. 